\title{
Comparación entre la realización de maquetas y la visualización para mejorar la alfabetización visual en anatomía humana en futuros docentes
}

\author{
Beatriz García Fernández ${ }^{1, a}$ Antonio Mateos Jiménez ${ }^{1, b}$ \\ ${ }^{1}$ Departamento de Pedagogía. Área de Didáctica de las Ciencias Experimentales. Universidad de Castilla- \\ La Mancha (España).

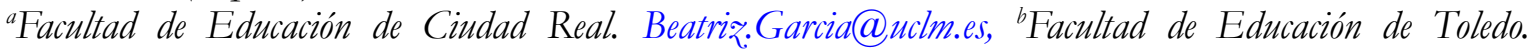 \\ Antonio.Mateos@uclm.es
}

[Recibido: 31 Diciembre 2017. Revisado: 9 Abril 2018. Aceptado: 12 Junio 2018]

\begin{abstract}
Resumen: La alfabetización visual es esencial para la formación docente en ciencias, especialmente en anatomía humana, cuya enseñanza y aprendizaje están apoyados en el uso de gráficos y requiere la adquisición de una visión espacial del cuerpo humano. Sin embargo, ha sido poco estudiada en maestros de Primaria y no existen investigaciones que evalúen la efectividad de métodos que permitan mejorarla. El presente trabajo, llevado a cabo con 194 maestros en su último año de formación, además de hacer un diagnóstico inicial de su nivel de alfabetización visual en anatomía humana, contrasta el impacto de la visualización frente a la realización de maquetas para mejorarla. Para ello se realizó un pretest y un postest consistentes en la representación de una sección transversal humana. Los resultados ponen de manifiesto una limitada alfabetización visual inicial vinculada a la producción de este tipo de secciones, y evidencian un mayor aprendizaje asociado a la realización de maquetas.
\end{abstract}

Palabras clave: Didáctica de la biología; Alfabetización visual; Formación docente; Representaciones anatómicas; Modelos anatómicos.

Comparison between the creation of models and visualization to enhance visual literacy in human anatomy in preservice teachers.

Abstract: Visual literacy is essential in science teacher training, especially in human anatomy. The teaching and learning processes of human anatomy are based on the use of graphics, and a spacial perception of the human body is required. Nevertheless, it has been little addressed, and there are no works of assessment of methodologies to improve it in Primary teacher training. This work has been carried out with 194 preservice teachers, and besides an initial diagnosis of the visual literacy skills in human anatomy, the impact of visualization and modelization of the human body to improve it are contrasted. The production of a cross section human diagram has been used as pre and post test. The results show limited skills in visual literacy related to the production of this type of sections, and a higher impact of producing scaled models on learning.

Keywords: Biology education; Visual literacy; Teacher training; Anatomy graphics; Anatomy models.

Para citar este artículo: García Fernández, B., Mateos Jiménez, A. (2018). Comparación entre la realización de maquetas y la visualización para mejorar la alfabetización visual en anatomía humana en futuros docentes. Revista Eureka sobre Enseñanza y Divulgación de las Ciencias 15(3), 3605. doi:10.25267/Rev_Eureka_ensen_divulg_cienc.2018.v15.i3.3605

\section{Introducción}

El material gráfico aparece frecuentemente en el ámbito académico y tiene un importante papel en la enseñanza de las ciencias, siendo mayor su presencia en esta que en otras áreas curriculares de la Educación Primaria (Coleman, McTigue y Smolkin 2011). Por ello, el aprendizaje de la dimensión visual se hace fundamental en la educación obligatoria (Eilam 2012; National Academies of Sciences, Engineering, and Medicine 2016). Los docentes deben estar formados en la alfabetización visual, entendida como una competencia para encontrar, interpretar, evaluar, utilizar y crear imágenes (Association of College and Research Libraries ACRL - 2011). En el ámbito concreto de las ciencias, los maestros han de ser capaces de

\author{
Revista Eureka sobre Enseñanza y Divulgación de las Ciencias \\ Universidad de Cádi\%. APAC-Eureka. ISSN: 1697-011X \\ bttp:/ / dx.doi.org/10.25267/Rev_Eureka_ensen_divulg_cienc.2018.v15.i3.3605 \\ http:/ / reuredc.uca.es
}


emplear diversos tipos de gráficos e incorporarlos a su docencia (Dimopoulos, Koulaidis y Sklaveniti 2013) como un requisito fundamental de la educación científica de nuestro siglo (McTigue y Flowers 2011).

\section{Fundamentación teórica}

La alfabetización visual es particularmente necesaria en biología (Rybarczyk 2011) pues las imágenes no solo se limitan a hacer más atractivo un libro, sino que contribuyen a un mejor aprendizaje (McTigue y Flowers 2011). La alfabetización visual es multidisciplinar y su aplicación y activación dependen del contexto (Avgerinou 2011), por lo que es preciso considerar, para su evaluación, requerimientos curriculares y el propio perfil del estudiante (Bowen 2017). La creación de imágenes y gráficos aparece recogida en los estándares educativos de ciencias (National Research Council 2012; 2013). Esto es debido a que las imágenes poseen una dimensión extralingüística (McTigue y Flowers 2011) y su uso presenta dificultades relacionadas con el contenido, el contexto y los códigos y convenciones para su representación (López-Manjón y Postigo 2014). Esta complejidad implica que el empleo de imágenes debe ser abordado desde las primeras etapas educativas (Postigo y López-Manjón 2012), lo que demanda docentes bien formados en esta competencia.

\section{La importancia de la comunicación visual en biología}

El aprendizaje de las ciencias está vinculado, desde una perspectiva semiótica, a una comunicación multimodal de los conceptos, siendo el modo visual especialmente importante para representar y comprender el conocimiento científico (Kress, Jewitt, Ogborn y Charalampos 2001). En ocasiones, el conocimiento científico puede ir asociado mayoritariamente a un modo exclusivo de representación, como es el caso del conocimiento sobre el cuerpo humano en Educación Primaria, representado tradicionalmente mediante vistas frontales de aparatos aislados (Pérez de Eulate, Llorente y Andrieu 1999; Carvalho, Silva y Clément 2007) e imágenes con escasos componentes visuales. Es poco frecuente en libros españoles la representación de, al menos, dos perspectivas distintas del mismo fenómeno, y de diferentes planos de la realidad representada (López-Manjón y Postigo 2014), todo ello pese a ser recomendable la representación en biología de diferentes perspectivas y cortes (Postigo y López-Manjón 2015).

Para aprender ciencias de modo efectivo, los estudiantes deben comprender diferentes representaciones de los conceptos y procesos científicos, y ser capaces de trasladarlos de una forma de representación a otra (Ainsworth 1999). En este sentido, el docente tiene el papel de evitar la dependencia entre el conocimiento científico y un único modo de representación, debiendo introducir otras formas de comunicarlo y proporcionar al alumnado estas oportunidades en el aula (Dimopoulos et al. 2003; Prain y Waldrip 2006). La razón de ello es el vínculo existente entre una mejor comprensión de los conceptos científicos y el reconocimiento de las relaciones entre diferentes modos de representarlos, siendo necesario, más allá de emplear distintos tipos de representaciones, centrar los esfuerzos en que los estudiantes sean capaces de integrar y representar el conocimiento aprendido mediante unas formas de representación en otras diferentes (Prain y Waldrip 2006). Este proceso, no exento de dificultad, ha de ser guiado por el profesorado (Prain y Waldrip 2006), lo que requiere formarles para que sean competentes en este sentido. En este contexto más amplio, y en el ámbito concreto del aprendizaje del cuerpo humano, los docentes deben saber guiar este proceso de creación por parte del alumnado de nuevas formas de representación diferentes a aquellas que están habituados a usar, de modo que se integre el conocimiento adquirido a través de diferentes representaciones (Ainsworth 1999), contribuyendo de este modo al 
desarrollo de la alfabetización visual en ciencias desde la escuela. El papel del docente ha de ser, además, el de filtro de la información visual a la que tanto docentes como estudiantes tienen acceso, pues aunque internet y otras fuentes de información permiten acceder a recursos de calidad en biología, en ocasiones estos recursos presentan errores que pueden trasladarse al alumnado (Donovan y Venville 2014).

El uso de las formas de representación ha de justificarse, además, por las demandas del contenido a enseñar (Jaipal 2010). En biología resulta de indudable interés el campo del cuerpo humano, donde las imágenes son esenciales en los libros de texto (López-Manjón y Postigo 2016), y en el que el conocimiento adquirido mediante vistas frontales de aparatos aislados, al integrarse en una única sección transversal, activa competencias relacionadas con el diseño (Felten 2008), la visión espacial y la síntesis (Cheng y Mix 2014).

En el ámbito del cuerpo humano, el uso único de vistas frontales de aparatos aislados presenta importantes limitaciones, pues este tipo de imágenes no permite mostrar cuestiones específicas como la posición relativa de los órganos de diferentes aparatos, y la escala en una tercera coordenada. Así, solo con este tipo de vistas no puede percibirse la posición relativa del esófago respecto de la tráquea, esencial para comprender el movimiento de la zona laringofaríngea e inferior que se percibe al tacto en el proceso de deglución, que podría generar la idea errónea en los escolares de que aquello que palpan al tragar es el esófago. Este tipo de imágenes tampoco muestra el volumen de estos órganos al solo aparecer representado el cuerpo en un sistema de coordenadas bidimensional, ni tampoco el interior y detalle de órganos vitales como los pulmones y el corazón, necesarios para comprender su funcionamiento. Por ejemplo: las vistas frontales no representan todas las características de la pleura, los bronquios secundarios y terciarios y las cavidades del corazón, cuyo conocimiento, más allá de ir vinculado a un saber meramente enciclopédico de estos órganos, va unido a la comprensión de cómo funcionan. En el caso de los pulmones, las secciones transversales permiten estudiar este nivel de detalle y conectar la estructura bronquial, ramificada y ocupando un importante volumen (en las vistas frontales se aprecian mayoritariamente como estructuras bidimensionales), con la existencia de los alvéolos pulmonares (microscópicos) para comprender realmente cómo se produce el intercambio de gases y cómo pasan de la atmósfera al torrente sanguíneo y viceversa. También permiten mejorar la comprensión sobre cómo los pulmones quedan protegidos frente a golpes y roces con la parte interna de la cavidad torácica, función que cumplen la pleura y su cavidad pleural, detalles que se aprecian en una sección transversal. Y, si bien la estructura interna del corazón suele mostrarse en secciones frontales, una vista transversal permite ver su posición relativa respecto a los pulmones y el volumen que ocupa al incluir un eje de coordenadas que no están habituados a manejar.

Destacamos que es común que tanto niños como maestros cometan errores en relación con la posición y el tamaño del corazón en esta tercera coordenada, siendo frecuente que lo ubiquen completamente centrado, detrás de los pulmones, o incluso al lado de la axila, y a una escala incorrecta (García Fernández y Ruiz-Gallardo 2017; Gatt y Saliba 2006; Ruiz-Gallardo, García Fernández y Mateos 2017) y con una estructura interna inadecuada (Bahar, Ozel, Prokop y Usak 2008). Las secciones transversales permiten visualizar el tronco pulmonar, de modo que se aprecia la conexión del corazón con el interior de los pulmones mediante la arteria y vena pulmonares y sus ramificaciones (en las vistas frontales no suelen verse estas conexiones ramificadas, y es frecuente que las arterias y venas pulmonares desaparezcan cuando se adentran en los pulmones).

Los cortes transversales permiten comparar el tamaño de las secciones de vasos tan importantes como las arterias aorta y pulmonar y las venas cavas y pulmonar, con otros vasos 
menores, incluso microscópicos, relacionando este aspecto con la cantidad de flujo de sangre en ellas y en sus ramificaciones, y con la presión sanguínea diferente en venas y en arterias, analizando en detalle las diferencias de grosor de las paredes de estos vasos. Mediante las secciones transversales se puede observar la vascularización del interior de órganos como el hígado, los riñones, el páncreas o los pulmones, lo cual permite comprender cómo se realizan las conexiones de estos órganos con el aparato circulatorio. Este tipo de secciones permite también cubrir el vacío de conocimiento reflejado en las secciones frontales de aparatos aislados con relación a los elementos existentes en el espacio entre los órganos de estos aparatos, que suele ser frecuentemente asociado por niños y maestros que han estudiado anatomía únicamente mediante secciones frontales a vasos sanguíneos y sangre como elementos "de relleno", en vez de asociarlos a la función de transporte (Ruiz-Gallardo et al., 2017; García Fernández y Ruiz-Gallardo, 2017), alejándose de un modelo científico completo de la función de nutrición.

\section{Modelos y modelización}

Un modelo científico es una representación abstracta y simplificada de un sistema o de un fenómeno que se centra en sus aspectos principales (Harrison y Treagust 2000). El proceso de construcción de un modelo (o modelización) se desarrolla mediante los razonamientos y experiencias concretas (Justi y Gilbert 2002) que pueden apoyarse en recursos como analogías (Acevedo-Díaz, García-Carmona, Aragón-Méndez y Oliva-Martínez 2017), proyecciones bidimensionales u objetos tridimensionales como maquetas (Bahamonde y Gómez Galindo 2016), ya que un modelo, pese a considerarse abstracto, va asociado a representaciones concretas para su comunicación (Buckley 2000).

El caso concreto del modelo de la función de nutrición es considerado, junto a los de las funciones de relación y reproducción, submodelos que integran el modelo de ser vivo (Bahamonde y Gómez Galindo 2016), parte esencial en el currículo de ciencias de Primaria (Real Decreto de Enseñanzas Mínimas 126/2014) y en la formación de maestros (MartínezChico, Jiménez Liso y López-Gay 2015). Los órganos, su localización, forma y escala también forman parte explícita del currículo de ciencias (Real Decreto de Enseñanzas Mínimas 126/2014), y su modelización constituye una parte del proceso aprendizaje de la función de nutrición (Bahamonde y Gómez Galindo 2016).

Generar un modelo mental de la función de nutrición presenta complejidades asociadas con la conexión de los procesos que se llevan a cabo en cada uno de los aparatos que participan en ella: digestivo, circulatorio, respiratorio y excretor (Núñez y Banet 1997). Es en este punto donde la realización de representaciones tridimensionales físicas del cuerpo humano (maquetas) ofrece ventajas didácticas, pues permite no solo representar y localizar las diferentes estructuras anatómicas, favoreciendo la construcción de un modelo mental tridimensional del cuerpo humano (Fančovičova y Prokop 2014), sino también hacer hincapié en representar las conexiones entre estos órganos y relacionar estas conexiones físicas con las conexiones entre los procesos que se llevan a cabo en los diferentes aparatos, huyendo así de un conocimiento anatómico desvinculado de los procesos que hacen posible la vida. También implica un proceso activo, lo que se vincula a un mejor aprendizaje en ciencias (Freeman et al. 2014; Gil 2014).

Representar la realidad modelizada constituye además un verdadero proceso de aprendizaje (Acevedo-Díaz et al. 2017) que permite acceder al modelo mental e intervenir en el proceso de enseñanza-aprendizaje (Bahamonde y Gómez Galindo 2016) mediante la detección de errores en los modelos físicos. La construcción del modelo mental y su representación en un modelo físico, requiere la adquisición previa de información, siendo las imágenes soportes semióticos 
útiles para adquirirla (Buckley 2000), por lo que los procesos de modelización y de visualización no pueden desligarse.

Saber interpretar correctamente imágenes anatómicas implica una complejidad y unas dificultades derivadas de la necesaria comprensión en tres dimensiones (3D) del cuerpo humano (Preece, Williams, Lam y Weller, 2013). La elaboración de secciones transversales precisa construir un modelo mental previo de la anatomía humana, y la visualización de imágenes en 2D y 3D es una estrategia didáctica que contribuye a ello, pues su empleo documentado en la formación de docentes, pese a ser muy escaso, muestra resultados positivos en el aprendizaje (De Jager 2017).

Por todo lo anteriormente referido, los maestros precisan de un adecuado nivel de alfabetización visual en anatomía que no siempre está presente y que se traduce en problemas en el aprendizaje de contenidos y en carencias en la visión espacial sobre el cuerpo humano (Ruiz-Gallardo et al. 2017).

\section{Objetivos}

Partiendo de la escasez de investigaciones que han evaluado la eficacia de estrategias orientadas a lograr la consecución, en los futuros docentes, de estándares de alfabetización visual en biología, y en concreto en un aspecto tan relevante en Educación Primaria como es la anatomía interna asociada a la nutrición, se plantean los siguientes objetivos:

Evaluar el nivel inicial de alfabetización visual vinculada a la creación de secciones anatómicas transversales humanas de maestros en formación.

- Contrastar la eficacia didáctica de la realización de maquetas frente al empleo de la visualización en dos y tres dimensiones del tronco humano para la mejora de la competencia en alfabetización visual asociada a la anatomía humana.

\section{Metodología}

\section{Diseño de la investigación}

La presente investigación aborda la comparación entre dos casos. Uno de ellos se apoya en el empleo de la visualización como recurso de aprendizaje, y el otro se basa en la modelización mediante la construcción de una maqueta del tronco humano. En la investigación se ha utilizado una prueba gráfica como pretest y postest.

\section{Muestra}

La muestra ha sido seleccionada mediante muestreo no probabilístico intencional y está constituida por estudiantes de cuarto curso del Grado de Maestro en Educación Primaria de la Universidad de Castilla-La Mancha (España). La muestra se divide en dos cohortes (1 y 2) correspondientes a dos cursos académicos consecutivos, empleando en cada uno de ellos métodos diferentes. La cohorte 1 se compone de 92 sujetos (30 varones y 62 mujeres; edad: $\overline{\mathrm{X}}=21,9$ años, DT $=1,5$ años) y la cohorte 2 de 102 sujetos (31 varones y 71 mujeres; edad: $\overline{\mathrm{X}}=22,6$ años, DT $=3,0$ años).

\section{Instrumento de recogida de datos}

Se ha utilizado como instrumento de recogida de datos, a modo de pretest y postest, una prueba gráfica con el siguiente enunciado: "Dibuja una sección transversal humana del tórax a nivel del pecho en la que aparezcan, al menos, los órganos implicados en la función de 
nutrición". Se eligió esta sección porque en ella aparecen órganos de tres de los cuatro aparatos implicados en la función de nutrición (respiratorio, circulatorio y digestivo), y permite trabajar la conexión entre los aparatos circulatorio y respiratorio). Durante la realización de la prueba se explicó la diferencia entre una sección frontal y otra transversal empleando como ejemplos un tocón de árbol y una rodaja de embutido. Tanto esta prueba como su instrumento de evaluación (Tabla 1) han sido utilizados anteriormente con maestros en formación (Ruiz-Gallardo et al. 2017) y con escolares de Primaria (García Fernández y RuizGallardo 2017) para evaluar la alfabetización visual en anatomía humana.

\section{Contexto didáctico}

Los estudiantes participantes cursaban la asignatura "El Medio Natural II: Biología, Geología y su Didáctica" de $4^{\circ}$ curso del Grado de Maestro en Educación Primaria. El programa de esta asignatura incluye la función de nutrición humana y se centra en revisar los principales contenidos que un maestro de Educación Primaria debe conocer de acuerdo al currículo oficial actual (Real Decreto de Enseñanzas Mínimas 126/2014), contenidos que, por otra parte, han estudiado previamente en varias ocasiones en Primaria y Secundaria. En el momento del comienzo de la investigación, la anatomía y fisiología de la función de nutrición había sido abordada en clase mediante secciones frontales de aparatos aislados. Los participantes habían cursado en $3^{\text {er }}$ curso la asignatura "Educación plástica y visual" única asignatura del plan de estudios en la que se aborda explícitamente el dibujo, pero sin tratar las representaciones anatómicas.

La secuencia didáctica empleada con la cohorte 1 comenzó con la realización del pretest y posterior reflexión sobre los errores cometidos más frecuentes. Para ello se acudió a la comparación de la sección dibujada por los estudiantes con una correcta, representándola, puesto que dibujar permite centrar la atención en los detalles (Davies 2010). Seguidamente se plantearon diversas preguntas, extraídas del trabajo exploratorio de Ruiz-Gallardo et al. (2017): A) Si has dibujado una sección frontal, ¿por qué lo has hecho? B) ¿Por qué has dibujado órganos que no aparecen a nivel del pecho? C) Si has dejado espacios en blanco en tu dibujo, ¿qué crees que hay en ese espacio? D) ¿Cómo permanecen los órganos en la posición en la que los has representado? ¿Se mueven de un sitio a otro por el espacio en blanco de tu dibujo? E) Si has representado las costillas completamente horizontales, ¿por qué lo has hecho? ¿Dónde empiezan y dónde acaban las costillas?. En las siguientes sesiones, los estudiantes pudieron acceder en la web a secciones transversales en 2D a distintos niveles (visualizadas desde el banco de recursos multimedia del Ministerio de Educación, Cultura y Deportes ${ }^{1}$ ), y frontales y 3D (visualizadas desde la web www.zygotebody.com), ya que el único empleo de secciones en 2D está asociado a una menor adquisición de los contenidos, y visualizar en 3D proporciona mejores resultados (Preece et al. 2013). La temporalización y secuencia seguida para la cohorte 1 fue la siguiente: pretest (1 hora), visualización, discusión y trabajo en el aula de vistas frontales y en $3 \mathrm{D}$ ( 2 horas) y transversales ( 2 horas), visualización de la conexión anatómica entre órganos, asociada a la conexión de procesos en la función de nutrición (2 horas), y postest al finalizar la asignatura, cuatro semanas tras la intervención (1 hora).

El método empleado en la cohorte 2 comenzó con la realización del pretest y posterior reflexión sobre los errores cometidos más frecuentes comparando el gráfico generado con una sec-

\footnotetext{
${ }^{1}$ Pueden consultarse en:

http://recursostic.educacion.es//bancoimagenes/ArchivosImagenes/DVD03/CD01/h16932_m.jpg http://recursostic.educacion.es//bancoimagenes/ArchivosImagenes/DVD03/CD01/h16917_m.jpg http:/ / recursostic.educacion.es//bancoimagenes/ArchivosImagenes/DVD03/CD01/h16929_m.jpg http://recursostic.educacion.es//bancoimagenes/ArchivosImagenes/DVD03/CD01/h16930_m.jpg http://recursostic.educacion.es//bancoimagenes/ArchivosImagenes/DVD03/CD01/h16928_m.jpg
} 
ción correcta, representando esta última para centrar la atención en los detalles y respondiendo a las mismas preguntas empleadas en la cohorte 1. Posteriormente, se propuso la creación de una maqueta del tronco humano en la que se incluyesen, al menos, los órganos implicados en la función de nutrición. Este modelo debía estar construido con materiales seguros, de sencilla manipulación y económicos, de modo que pudiesen emplearlo en Primaria. El modelo debía ir acompañado de un material didáctico que reflejase la función de los diferentes órganos representados, conexión entre ellos y entre procesos de los distintos aparatos, y discusión de las limitaciones que presentaba. La construcción de la maqueta, material didáctico y análisis de limitaciones se llevó a cabo fuera del aula, y requirió de una fase de búsqueda activa de información por parte del alumnado, al que se preguntó posteriormente por el tiempo dedicado a la realización de esta actividad. La temporalización y secuencia seguida fue la siguiente: pretest (1 hora), trabajo autónomo para realizar las maquetas (Figura 1) y discutir sus limitaciones en grupos de 4-5 personas (10,5 horas dedicadas de media por persona, DT $=4,7$ horas), evaluación formativa en el aula de los trabajos (1 hora), y postest al finalizar la asignatura, cuatro semanas tras la intervención (1 hora).

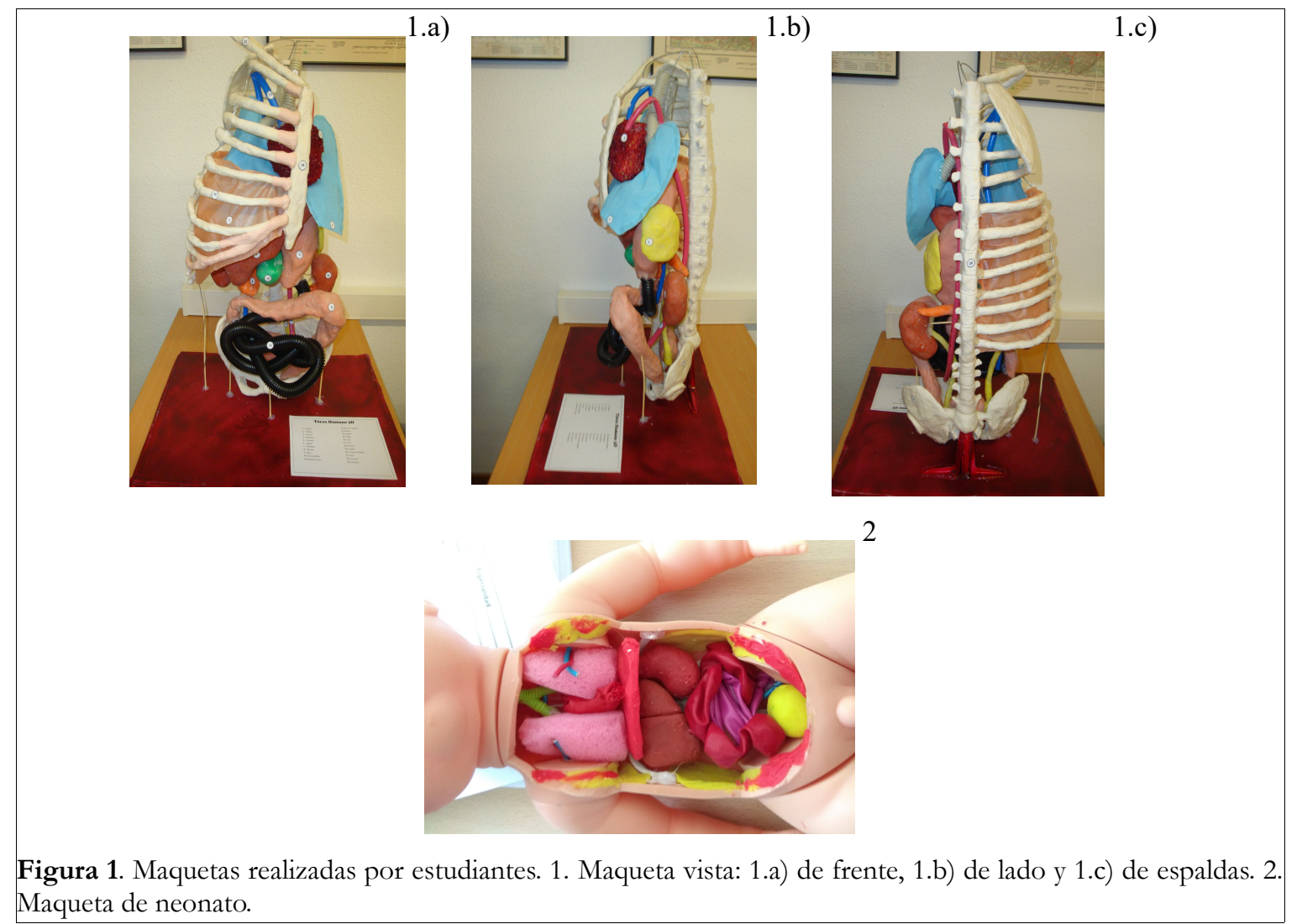

\section{Procedimientos de análisis de datos}

El instrumento que se ha empleado para evaluar la prueba gráfica del pretest y el postest recoge criterios de la Association of College and Research Libraries (2011) que definen la competencia en alfabetización visual. Estos criterios se han adaptado para esta prueba (Tabla 1) y validado con maestros en formación (Ruiz-Gallardo et al. 2017). Concretamente, se han considerado los elementos representados en la sección (A), distribución (B), escala (C), sencillez de interpretación (D), tamaño (E), etiquetado (F) y adecuación a estándares de formación docente $(\mathrm{G})$ : 
A - 0,04 puntos por cada órgano correcto representado con máximo de 0,2 puntos.

B - 0,15 puntos si no presenta errores, 0,1 puntos cuando hay un error, 0,05 puntos cuando hay dos errores, 0 puntos cuando hay 3 o más errores.

C - 0,15 puntos si dibuja cinco o más órganos a escala correcta, 0,1 puntos si dibuja cuatro órganos a escala correcta, 0,075 puntos si dibuja tres, 0,05 puntos si dibuja dos, 0,025 puntos si dibuja uno, 0 puntos si no dibuja ninguno a escala correcta.

D - 0,1 puntos si no hay elementos ininteligibles, 0,05 puntos si hay un elemento ininteligible, y 0 puntos si hay más de un elemento ininteligible.

E - 0,1 puntos si el tamaño de la sección permite representar detalles, 0 si es demasiado pequeña y no permite representar detalles. Puede puntuarse linealmente si solo permite representar algunos detalles, pero no todos (por ejemplo, si permite representar los bronquios, pero no la pleura: 0,05 puntos).

F - 0,04 puntos por órgano correcto etiquetado con máximo de 0,2 puntos.

$\mathrm{G}$ - Detalles de los principales órganos representados que han de mostrarse en la escuela: cavidades del corazón (0,05 puntos), pleura ( 0,05 puntos) y bronquios secundarios y terciarios ( 0,05 puntos), con un máximo de 0,15 puntos.

Para el análisis de datos se empleó el software SPSS v. 23. Se aplicó el test de KolmogorovSmirnov a las puntuaciones obtenidas en la prueba gráfica y, si bien las variables son de tipo escalar, al no satisfacerse el criterio de normalidad $(p<0,05)$, se empleó estadística no paramétrica (Field 2009): U de Mann Withney para muestras independientes y prueba de rangos de Wilcoxon para muestras relacionadas. El análisis se complementó con el tamaño del efecto y estadística descriptiva.

\section{Resultados}

Se han comparado las calificaciones obtenidas en ambas cohortes en el pretest a través de un análisis transversal. La prueba $U$ de Mann Withney muestra que inicialmente no existen diferencias estadísticamente significativas entre ellas en la puntuación general $(p>0,05)$. Tan solo existen diferencias significativas en los criterios B ( $p=0,025), \mathrm{E}(p=0,027)$ y $\mathrm{G}(p=0,003)$, aunque puntuando muy bajo ambas cohortes en los tres casos (Tabla 1$)$.

Los resultados muestran la limitada competencia en producción de secciones transversales que tiene inicialmente el alumnado (Tabla 1). Algunas de las secciones elaboradas en el pretest que recogen los errores más frecuentes se muestran en la Figura 2. Los resultados de la prueba de Wilcoxon en todas las dimensiones evaluadas (Tabla 1) muestran que existen diferencias significativas entre pre y postest para ambas cohortes $(p<0,05)$. Los resultados de la prueba tamaño del efecto (Tabla 1) indican que la intervención basada en la realización de maquetas (cohorte 2) tiene un mayor impacto que la basada en la visualización (cohorte 1). De la estadística descriptiva aplicada a las diferentes dimensiones (Tabla 1) también se desprende este mayor impacto en el aprendizaje en la cohorte 2 , si bien se han observado mejoras en ambas cohortes.

Se han comparado las destrezas (Tabla 1) en un análisis transversal en el postest, encontrándose que la cohorte 2 (en la que se han realizado maquetas) puntúa significativamente mejor tanto a nivel global como en todas las destrezas parciales consideradas ( $p=0,000$ en todos los casos). Ello implica que, incluso en las tres dimensiones en las que inicialmente la cohorte 1 (en la que se ha empleado la visualización) puntuaba significativamente mejor, al finalizar las intervenciones, la cohorte 2 puntúa significativamente mejor que la 1. 
El análisis de las características gráficas de las secciones (Tabla 2) y de los principales elementos representados (Tabla 3) que han dado lugar a las puntuaciones anteriores, evidencia que, aunque en ambas cohortes se producen mejoras estadísticamente significativas, la realización de maquetas se traduce en mejores resultados al finalizar las intervenciones. Un análisis estadístico transversal de los resultados comparando ambas cohortes en el postest, muestra que la cohorte 2 presenta mejores resultados que la 1 de manera estadísticamente significativa con relación a la disminución de amplios espacios vacíos, representación de elementos que no deberían aparecer, distribución razonable de órganos y la representación de estos a la escala correcta (ver ejemplos de secciones con correcta distribución espacial en la Figura 3), la presencia de detalles (ver ejemplos de secciones con detalles en la Figura 3) y la reducción de la presencia de elementos ininteligibles $(p<0,05)$. También la cohorte 2 representa de una forma estadísticamente significativa más órganos correctos (ver ejemplos en la Figura 3), concretamente el corazón, los pulmones, el esófago, la vena cava, la arteria aorta, las venas y arterias pulmonares, las venas y arterias representadas de modo genérico, la columna vertebral, la médula espinal, las costillas y el esternón $(p<0,05)$, y aquellos en los que las diferencias no son estadísticamente significativas (piel, musculatura torácica y bronquios; $p>0,05)$, la cohorte 2 también presenta mejores resultados.

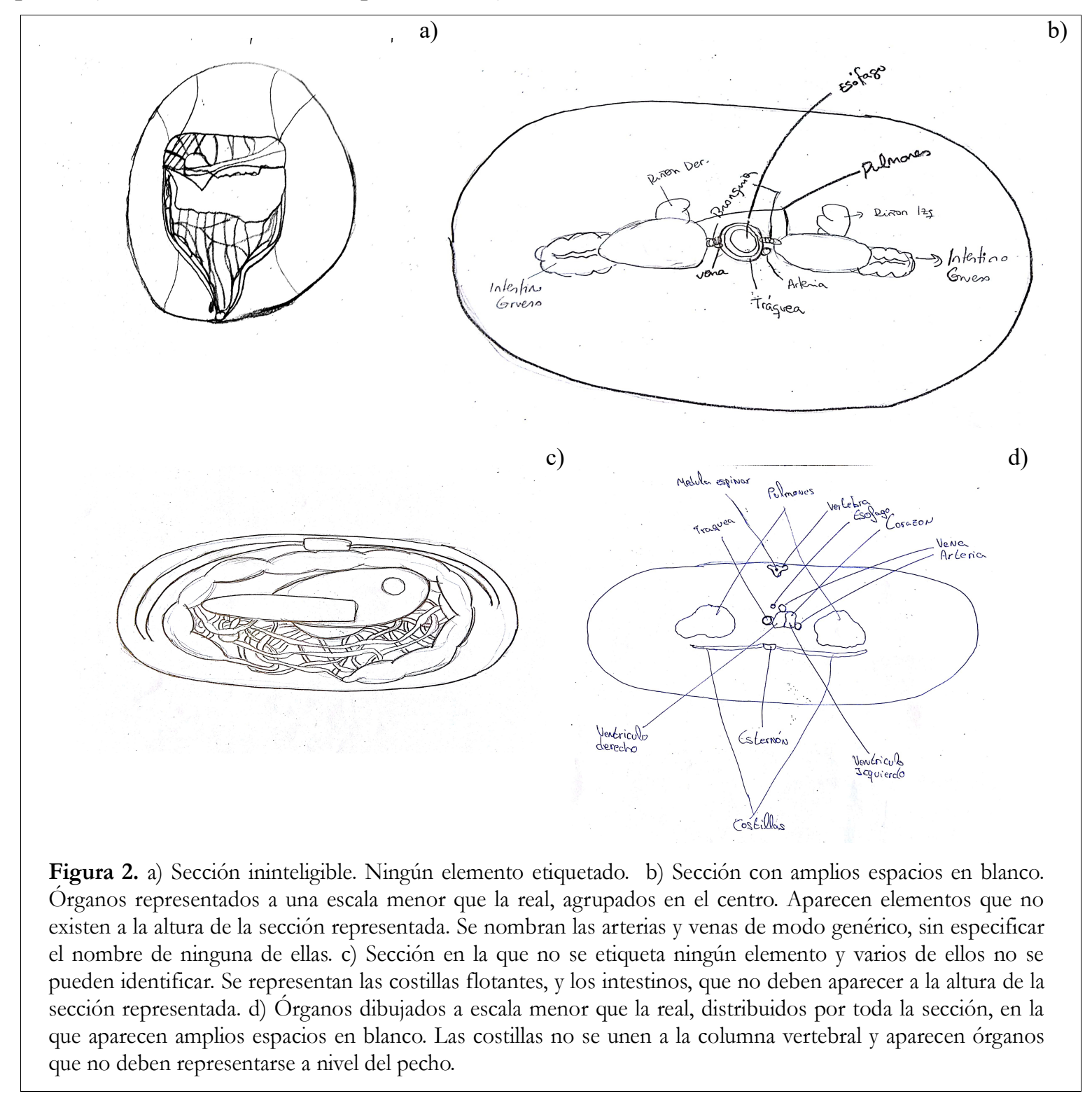


Tabla 1. Criterios de evaluación para la prueba gráfica (Fuente: Ruiz-Gallardo et al. 2017; García Fernández y Ruiz-Gallardo 2017). Puntuaciones, significación y tamaño del efecto obtenidos en el pretest y en el postest para cada cohorte.

\begin{tabular}{|c|c|c|c|c|c|c|c|c|c|}
\hline \multirow[b]{2}{*}{$\begin{array}{l}\text { Criterios de } \\
\text { evaluación }\end{array}$} & \multirow[b]{2}{*}{$\begin{array}{l}\text { Rango } \\
\text { valor } \\
\text { parcial }\end{array}$} & \multicolumn{4}{|c|}{ Cohorte $1(\mathrm{~N}=92)$} & \multicolumn{4}{|c|}{ Cohorte $2(\mathrm{~N}=102)$} \\
\hline & & Pretest & Postest & $p$ & $\begin{array}{c}\text { Tamaño } \\
\text { del efecto }\end{array}$ & Pretest & Postest & $p$ & $\begin{array}{c}\text { Tamaño } \\
\text { del efec- } \\
\text { to }\end{array}$ \\
\hline $\begin{array}{l}\text { A. La sección } \\
\text { contiene todos } \\
\text { los elementos } \\
\text { estudiados (al } \\
\text { menos corazón, } \\
\text { pulmones, esó- } \\
\text { fago, vena cava } \\
\text { y arteria aorta). }\end{array}$ & $0-0,20$ & $\begin{array}{c}\overline{\mathbf{X}}: 0,073 \\
\text { DT: } 0,075\end{array}$ & $\begin{array}{c}\overline{\mathbf{X}}: 0,123 \\
\text { DT: } 0,058\end{array}$ & 0,000 & $-0,571$ & $\begin{array}{c}\bar{X}: 0,060 \\
\text { DT: } 0,041\end{array}$ & \begin{tabular}{|c}
$\overline{\mathbf{X}}: 0,174$ \\
DT: 0,037
\end{tabular} & 0,000 & $-0,845$ \\
\hline $\begin{array}{l}\text { B. Adecuada } \\
\text { distribución de } \\
\text { órganos y del } \\
\text { espacio dentro } \\
\text { de la sección. }\end{array}$ & $0-0,15$ & $\begin{array}{c}\overline{\mathbf{x}}: 0,010 \\
\text { DT: } 0,029\end{array}$ & $\begin{array}{c}\overline{\mathbf{X}}: 0,062 \\
\text { DT: } 0,057\end{array}$ & 0,000 & $-0,616$ & $\begin{array}{c}\overline{\mathbf{x}}: 0,002 \\
\text { DT: } 0,011\end{array}$ & $\begin{array}{c}\overline{\mathrm{X}}: 0,107 \\
\text { DT:0,048 }\end{array}$ & 0,000 & $-0,850$ \\
\hline $\begin{array}{l}\text { C. Los órganos } \\
\text { aparecen repre- } \\
\text { sentados a una } \\
\text { escala adecua- } \\
\text { da. }\end{array}$ & $0-0,10$ & $\begin{array}{c}\overline{\mathbf{X}}: 0,012 \\
\text { DT: } 0,026\end{array}$ & $\begin{array}{c}\overline{\text { X: }} 0,064 \\
\text { DT: } 0,042\end{array}$ & 0,000 & $-0,728$ & $\begin{array}{l}\bar{X}:: 0,007 \\
\text { DT:0,017 }\end{array}$ & \begin{tabular}{|c}
$\overline{\mathbf{X}}: 0,093$ \\
DT:0,015
\end{tabular} & 0,000 & $-0,897$ \\
\hline $\begin{array}{l}\text { D. La sección } \\
\text { es sencilla de } \\
\text { interpretar. }\end{array}$ & $0-0,10$ & $\begin{array}{c}\text { X̄: }, 0,003 \\
\text { DT: } 0,013\end{array}$ & $\begin{array}{c}\overline{\text {X}}: 0,042 \\
\text { DT: } 0,045\end{array}$ & 0,000 & $-0,610$ & $\begin{array}{c}\overline{\mathrm{X}}: 0,005 \\
\text { DT:0,019 }\end{array}$ & \begin{tabular}{|c|}
$\bar{X}: 0,069$ \\
DT:0,041
\end{tabular} & 0,000 & $-0,784$ \\
\hline $\begin{array}{l}\text { E. El tamaño } \\
\text { de la sección } \\
\text { permite que } \\
\text { aparezcan re- } \\
\text { presentados los } \\
\text { detalles que han } \\
\text { de mostrarse al } \\
\text { niño. }\end{array}$ & $0-0,10$ & $\begin{array}{c}\overline{\mathbf{X}}: 0,006 \\
\text { DT: } 0,019\end{array}$ & $\begin{array}{c}\overline{\text { X}}: 0,047 \\
\text { DT: } 0,041\end{array}$ & 0,000 & $-0,681$ & $\begin{array}{c}\bar{X}: 0,003 \\
\text { DT:0,018 }\end{array}$ & \begin{tabular}{|l}
$\overline{\mathrm{X}}: 0,075$ \\
DT:0,037
\end{tabular} & 0,000 & $-0,832$ \\
\hline $\begin{array}{l}\text { F. Todos los } \\
\text { elementos en la } \\
\text { sección están } \\
\text { etiquetados cla- } \\
\text { ra y correcta- } \\
\text { mente. }\end{array}$ & $0-0,20$ & $\begin{array}{c}\overline{\text {X}}: 0,068 \\
\text { DT: } 0,069\end{array}$ & $\begin{array}{c}\overline{\text { X}}: 0,148 \\
\text { DT: } 0,079\end{array}$ & 0,000 & $-0,689$ & $\begin{array}{c}\overline{\mathbf{X}}: 0,064 \\
\text { DT:0,059 }\end{array}$ & \begin{tabular}{|c} 
X्र: 0,192 \\
DT:0,026
\end{tabular} & 0,000 & $-0,807$ \\
\hline $\begin{array}{l}\text { G. La sección } \\
\text { alcanza los es- } \\
\text { tándares ade- } \\
\text { cuados para un } \\
\text { maestro (al me- } \\
\text { nos incluye la } \\
\text { pleura, cavida- } \\
\text { des del corazón } \\
\text { y bronquios } \\
\text { primarios y se- } \\
\text { cundarios) que } \\
\text { han de mostrar- } \\
\text { se al niño. }\end{array}$ & $0-0,15$ & $\begin{array}{c}\overline{\text { X}}: 0,023 \\
\text { DT: } 0,054\end{array}$ & $\begin{array}{c}\overline{\text { X}}: 0,055 \\
\text { DT: } 0,050\end{array}$ & 0,000 & $-0,444$ & $\begin{array}{c}\overline{\mathrm{X}}: 0,002 \\
\text { DT:0,011 }\end{array}$ & $\begin{array}{c}\bar{X}: 0,085 \\
\text { DT: } 0,050\end{array}$ & 0,000 & $-0,812$ \\
\hline $\begin{array}{l}\text { Media de las } \\
\text { puntuaciones } \\
\text { totales de la ta- } \\
\text { rea (sobre 1). }\end{array}$ & 1 & $\begin{array}{c}\overline{\mathbf{X}}: 0,178 \\
\text { DT: } 0,159\end{array}$ & $\begin{array}{c}\overline{\mathbf{X}}: 0,542 \\
\text { DT: } 0,302\end{array}$ & 0,000 & $-0,599$ & $\begin{array}{c}\overline{\mathrm{X}}: 0,143 \\
\text { DT: } 0,126\end{array}$ & \begin{tabular}{|c|}
$\mathbf{X}: 0,792$ \\
DT: 0,189
\end{tabular} & 0,000 & $-0,868$ \\
\hline
\end{tabular}



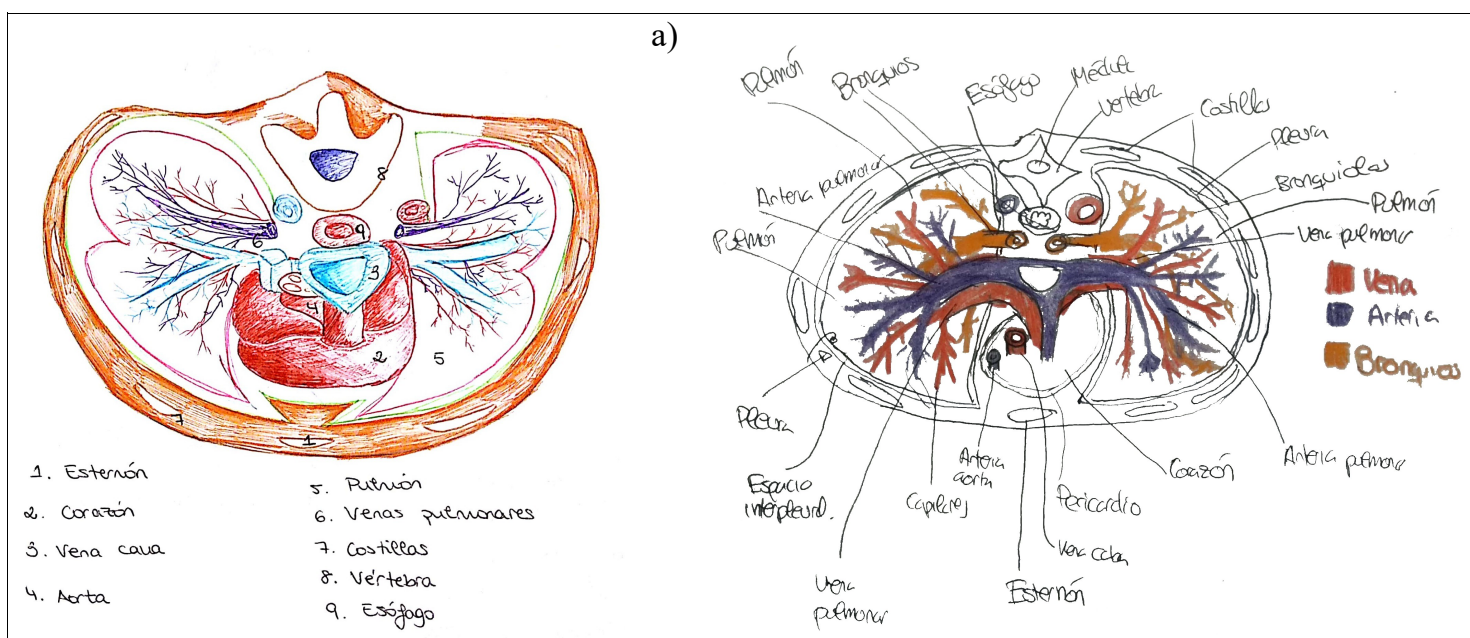

b)

Figura 3. a) Sección con distribución razonable de órganos y elementos sin etiquetar. El corazón aparece en el centro, aunque el pulmón izquierdo es de menor tamaño que el derecho. b) Sección con adecuada distribución de órganos. Incluye detalles y todos los elementos aparecen etiquetados.

Tabla 2. Resultados del análisis de la dimensión gráfica de las secciones.

\begin{tabular}{|l|c|c|c|c|c|c|}
\hline & \multicolumn{3}{|c|}{ Cohorte 1 (N=92) } & \multicolumn{3}{c|}{ Cohorte 2 (N=102) } \\
\cline { 2 - 7 } & Pretest & Postest & p & Pretest & Postest & p \\
\hline Presencia de amplios espacios vacíos & $44(47,8 \%)$ & $9(9,8 \%)$ & 0,000 & $38(19 \%)$ & $2(1,8 \%)$ & 0,000 \\
\hline $\begin{array}{l}\text { Presencia de elementos que no deben } \\
\text { aparecer a la altura del pecho }\end{array}$ & $59(64,1 \%)$ & $18(19,6 \%)$ & 0,000 & $22(19,5 \%)$ & $2(1,8 \%)$ & 0,000 \\
\hline $\begin{array}{l}\text { Organos agrupados en el centro de la } \\
\text { sección }\end{array}$ & $24(26,1 \%)$ & $2(2,2 \%)$ & 0,000 & $11(9,7 \%)$ & $6(5,3 \%)$ & 0,014 \\
\hline $\begin{array}{l}\text { Representación de una sección frontal } \\
\text { completa }\end{array}$ & $9(9,8 \%)$ & $0(0,0 \%)$ & 0,003 & $17(15,0 \%)$ & $0(0,0 \%)$ & 0,000 \\
\hline $\begin{array}{l}\text { Representación de una sección transver- } \\
\text { sal que incluye órganos representados en } \\
\text { sección frontal }\end{array}$ & $13(14,1 \%)$ & $3(3,3 \%)$ & 0,012 & $19(16,8 \%)$ & $0(0,0 \%)$ & 0,000 \\
\hline $\begin{array}{l}\text { Órganos aislados y distribuidos por toda } \\
\text { la sección }\end{array}$ & $13(14,1 \%)$ & $4(4,3 \%)$ & 0,013 & $7(6,2 \%)$ & $0(0,0 \%)$ & 0,008 \\
\hline $\begin{array}{l}\text { Representación de elementos sin etique- } \\
\text { tar }\end{array}$ & $38(41,3 \%)$ & $30(32,6 \%)$ & 0,228 & $27(23,9 \%)$ & $46(40,7 \%)$ & 0,005 \\
\hline $\begin{array}{l}\text { Representación de órganos a una escala } \\
\text { menor }\end{array}$ & $38(41,3 \%)$ & $7(7,6 \%)$ & 0,000 & $35(31,0 \%)$ & $1(0,9 \%)$ & 0,000 \\
\hline $\begin{array}{l}\text { Distribución razonable de órganos en la } \\
\text { sección }\end{array}$ & $1(1,1 \%)$ & $34(37,0 \%)$ & 0,000 & $0(0,0 \%)$ & $81(71,7 \%)$ & 0,000 \\
\hline $\begin{array}{l}\text { Presencia de más de un elemento ininte- } \\
\text { ligible }\end{array}$ & $85(92,4 \%)$ & $46(50,0 \%)$ & 0,000 & $96(94,1 \%)$ & $23(22,6 \%)$ & 0,000 \\
\hline $\begin{array}{l}\text { Sección con un tamaño que no permite } \\
\text { la representación de detalles }\end{array}$ & $80(87,0 \%)$ & $27(29,3 \%)$ & 0,000 & $92(90,2 \%)$ & $14(13,7 \%)$ & 0,000 \\
\hline
\end{tabular}


Tabla 3. Resultado del análisis de los elementos correctos representados en las secciones.

\begin{tabular}{|l|c|c|c|c|c|c|}
\hline \multirow{2}{*}{ Elementos } & \multicolumn{3}{|c|}{ Cohorte 1 (N=92) } & \multicolumn{3}{c|}{ Cohorte 2 (N=102) } \\
\cline { 2 - 7 } & Pretest & Postest & $\boldsymbol{p}$ & Pretest & Postest & $\boldsymbol{p}$ \\
\hline Corazón & $44(47,8 \%)$ & $79(85,9 \%)$ & 0,000 & $51(45,1 \%)$ & $102(100 \%)$ & 0,000 \\
\hline Pulmones & $57(62,0 \%)$ & $80(87,0 \%)$ & 0,000 & $75(66,4 \%)$ & $101(89,4 \%)$ & 0,000 \\
\hline Esófago & $38(41,3 \%)$ & $66(71,7 \%)$ & 0,000 & $23(20,4 \%)$ & $93(82,3 \%)$ & 0,000 \\
\hline Vena cava & $4(4,3 \%)$ & $27(29,3 \%)$ & 0,000 & $2(1,8 \%)$ & $67(59,3)$ & 0,000 \\
\hline Arteria aorta & $7(7,6 \%)$ & $30(32,6 \%)$ & 0,000 & $4(3,5 \%)$ & $80(70,8 \%)$ & 0,000 \\
\hline Vena pulmonar & $1(1,1 \%)$ & $28(30,4 \%)$ & 0,000 & $11(9,7 \%)$ & $61(54,0 \%)$ & 0,000 \\
\hline Arteria pulmonar & $0(0,0 \%)$ & $28(30,4 \%)$ & 0,000 & $11(9,7 \%)$ & $55(48,7 \%)$ & 0,000 \\
\hline Arterias y venas (genérico) & $8(8,7 \%)$ & $26(28,3 \%)$ & 0,000 & $12(10,6 \%)$ & $8(7,1 \%)$ & 0,317 \\
\hline Columna vertebral & $30(32,6 \%)$ & $72(78,3 \%)$ & 0,000 & $16(14,2 \%)$ & $100(88,5 \%)$ & 0,000 \\
\hline Médula espinal & $2(2,2 \%)$ & $22(23,9 \%)$ & 0,000 & $1(0,9 \%)$ & $80(70,8 \%)$ & 0,000 \\
\hline Costillas & $30(32,6 \%)$ & $55(59,8 \%)$ & 0,000 & $18(15,9 \%)$ & $93(82,3 \%)$ & 0,000 \\
\hline Esternón & $9(9,8 \%)$ & $66(71,7 \%)$ & 0,000 & $4(3,5 \%)$ & $93(82,3 \%)$ & 0,000 \\
\hline Piel & $0(0,0 \%)$ & $1(1,1 \%)$ & 0,317 & $2(1,8 \%)$ & $5(4,4 \%)$ & 0,257 \\
\hline Musculatura torácica & $1(1,1 \%)$ & $3(3,3 \%)$ & 0,317 & $1(0,9 \%)$ & $11(9,7 \%)$ & 0,004 \\
\hline Bronquios & $10(10,9 \%)$ & $48(52,2 \%)$ & 0,000 & $3(2,7 \%)$ & $60(53,1 \%)$ & 0,000 \\
\hline
\end{tabular}

\section{Discusión}

La alfabetización visual, esencial en la educación obligatoria y universitaria, se aplica de forma distinta según el contexto académico. En el caso de la formación docente en ciencias, los maestros deben ser competentes para enseñar a sus alumnos a interpretar y crear diagramas visuales, puesto que ello aparece incluido dentro de los estándares educativos de ciencias. En particular, en anatomía humana, los maestros han de ser capaces de crear y guiar el proceso de creación en su alumnado de diagramas visuales que impliquen trasladar información entre diferentes tipos de representaciones (Prain y Waldrip 2006), involucrando la visión espacial localizando los órganos en un tercer eje de coordenadas poco frecuente en libros de texto y representándolos de acuerdo a su forma y escala aproximadas - y síntesis - integrando el conocimiento aprendido en vistas frontales de aparatos aislados en un solo diagrama (Ainsworth 1999).

Sin embargo, los resultados de este trabajo evidencian que los maestros en formación de la muestra no poseen desarrollada adecuadamente esta competencia (pretest inferior a 0,18 sobre 1), presentando deficiencias en todas las dimensiones estudiadas. Estos resultados coinciden con trabajos previos (Ruiz-Gallardo et al. 2017; Ruiz-Gallardo, Valdés, Sáez y Roldán 2012) en los que se detectaron errores similares en maestros, pese a que todos los alumnos han estudiado anatomía en varias ocasiones en la educación obligatoria, si bien podría explicarse por el hecho de que los libros de texto suelen poseer limitaciones en la variedad de tipos de secciones y perspectivas (Postigo y López-Manjón 2015) con predominancia de vistas frontales de aparatos aislados (Pérez de Eulate et al. 1999). Todo ello viene a señalar la necesidad de diseñar, aplicar y evaluar métodos docentes que permitan mejorar esta competencia.

El empleo del método basado en la visualización produce mejoras estadísticamente significativas en todas las dimensiones estudiadas y se reducen los errores más frecuentes, aumentando el número de elementos correctamente representados. Estos resultados son coherentes con los obtenidos por Brenton et al. (2007) empleando secciones transversales, y también por De Jager (2017) en un estudio de caso, quien empleó la visualización tridimensional junto a secciones longitudinales y transversales en la formación de docentes de Secundaria de la especialidad de biología con resultados favorables. Sin embargo, aunque se producen mejoras significativas, la puntuación media obtenida es de apenas cinco sobre diez. 
Las destrezas en la cohorte 1 que obtienen menor puntuación (postest) son aquellas vinculadas con la visión espacial, y la que mayor puntuación obtiene es la que tiene que ver con el etiquetado. Ello puede deberse a que durante las sesiones de aula se trabajó específicamente el etiquetado, relacionándolo con la interpretación de la imagen. Sin embargo, en la representación correcta de órganos, su distribución y escala obtienen puntuaciones más bajas que la cohorte 2 , seguramente debido a que requieren la construcción de un modelo mental en 3D que entraña dificultades (Preece et al. 2013), siendo estas dimensiones en las que este método muestra más debilidad. Estos resultados están en la línea de los obtenidos por Biasutto, Caussa y Criado (2006), quienes evidenciaron que una aproximación meramente digital está asociada a un conocimiento anatómico pobre.

La realización de maquetas produce también mejoras estadísticamente significativas tanto en la puntuación total como en las dimensiones parciales evaluadas, y presenta mejores resultados que la visualización de imágenes en todas las dimensiones estudiadas, como revelan los resultados del análisis transversal de los datos. Este método también produce un mayor aumento de las respuestas correctas asociadas a órganos representados y de las destrezas gráficas. Pese a que las maquetas generadas tienen menos detalle que las imágenes visualizadas en la cohorte 1, su realización produce un mayor impacto en el aprendizaje, como revelan los resultados tanto descriptivos como del tamaño del efecto. Los resultados de la estadística descriptiva y análisis transversal referentes a la dimensión gráfica de las secciones (Tabla 3), evidencian mejores resultados para la cohorte 2. Únicamente en la cohorte 1 es menor el porcentaje de estudiantes que presenta elementos sin etiquetar, aunque ello se debe a que las secciones de la cohorte 2 incluyen más elementos, como muestran los resultados de la tabla 3 y es más frecuente que alguno de ellos no se etiquete. En relación a los elementos representados, la realización de maquetas también presenta mejores resultados. Tan solo en la representación de arterias y venas, de modo genérico, la cohorte 1 obtiene mejores resultados, aunque ello se debe a que en la cohorte 2 es mayor el porcentaje de estudiantes que nombra arterias y venas concretas.

Estos resultados evidencian que la realización de maquetas supone un mayor desarrollo en las destrezas vinculadas tanto al conocimiento de los elementos presentes en el cuerpo como a la visión tridimensional de los mismos frente a la visualización. Estos resultados siguen la línea de los obtenidos por Fančovičova y Prokop (2014), quienes sostienen que la realización de modelos físicos tridimensionales facilita la adquisición del conocimiento anatómico en tres dimensiones. Asimismo, coinciden con los resultados del trabajo de Preece et al. (2013) que señalan que el empleo de modelos físicos produce una mejor comprensión tridimensional de la información esencial para la construcción de secciones transversales. Esta visión de conjunto contribuye a un aprendizaje que integre los órganos de diferentes aparatos y sus conexiones, propiciando la comprensión de su funcionamiento y huyendo de un conocimiento fragmentado del cuerpo humano (Núñez y Banet 1997).

Los resultados obtenidos mediante la realización de maquetas, mejores que aquellos obtenidos mediante la visualización, pueden deberse, por otro lado, a una participación más activa del alumnado. Los estudiantes de la segunda cohorte debían recopilar información y sintetizarla en una maqueta, mientras que los estudiantes de la primera cohorte debían visualizar información que se les había proporcionado. Aunque en ambos casos se pretendía que se visualizase activamente, integrando la información en un modelo mental tridimensional, la construcción de una maqueta supone un proceso de aprendizaje más activo, e implica un mayor esfuerzo mental y activación del pensamiento por parte del alumno, y todo ello se vincula a un mejor aprendizaje (Freeman et al. 2014; Gil 2014). Asimismo, pueden deberse estos mejores resultados a una mayor dedicación de tiempo al trabajo autónomo, por lo que se 
propone como futura línea de trabajo la identificación de dificultades y distribución de la carga de trabajo que permitan caracterizar mejor esta actividad.

Destacamos que las maquetas generadas por los estudiantes no tienen tanto detalle como las correspondientes imágenes visualizadas, lo cual puede ser favorable ya que permite centrar el interés en las cuestiones y detalles importantes para el nivel de conocimiento que ha de poseer un maestro y que aparecen explícitamente en el currículo español de Primaria (forma, tamaño, y localización de órganos y las conexiones anatómicas y fisiológicas entre ellos), de modo que se contribuya a la adquisición de un correcto modelo mental de la función de nutrición. Además, el hecho de que sean modelos realizados con materiales seguros, baratos y fácilmente manipulables, permite aprender una estrategia que puede adaptarse al currículo de los diferentes cursos de Primaria y con mejores resultados que la presentación tradicionalmente empleada en los libros de texto, construida a partir de vistas frontales de aparatos aislados.

\section{Conclusiones}

Los resultados de este trabajo evidencian el escaso nivel de alfabetización visual relacionada con la anatomía humana de maestros en formación, pese a ser un contenido fundamental en el currículo escolar, lo cual justifica la necesidad de diseñar, aplicar y evaluar estrategias que permitan corregir estas carencias.

Aunque los dos métodos empleados implican mejoras significativas, la realización de una maqueta del tronco humano presenta un mayor impacto en la alfabetización visual en anatomía humana que la mera visualización de imágenes, tanto en las dimensiones relacionadas con la representación de elementos como en aquellas vinculadas con la visión espacial. Se destaca, además, que este método puede ser adaptado por los maestros a los diferentes cursos de Primaria con distintos grados de dificultad y detalle, con lo que no solo se convierte en una forma de mejorar el aprendizaje de contenidos anatómicos y la visión espacial, sino que además puede representar un recurso didáctico flexible para el docente.

Señalamos, como limitación, que es un estudio de caso en un contexto académico concreto y que ha de ampliarse la investigación a otros contextos y muestras. Asimismo, aunque se ha trabajado de manera explícita en ambas secuencias didácticas la conexión de los procesos que tienen lugar en los aparatos implicados en la función de nutrición, no se ha evaluado esta cuestión, pues escapaba a los objetivos de este trabajo y se plantea como centro de interés de pró ximos estudios.

\section{Referencias}

Acevedo-Díaz, J.A., García-Carmona, A., Aragón-Méndez, M.M., Oliva-Martínez, J.M. (2017) Modelos científicos: significado y papel en la práctica científica. Revista Científica 30 (3), 155-166. Doi: 10.14483/23448350.12288

Ainsworth, S. (1999) The functions of multiple representations. Computers \& education 33 (2-3), 131-152. Doi: 10.1016/S0360-1315(99)00029-9

Association of College and Research Libraries (2011) ACRL visual literacy competency standards for higher education. Chicago, Illinois: American Library Association. Recuperado de: http://www.ala.org/acrl/standards/visualliteracy

Avgerinou, M. (2011) Toward a Cohesive Theory of Visual Literacy. Journal of Visual Literacy 30 (2), 1-19. Doi: 10.1080/23796529.2011.11674687 
Bahamonde, N., Gómez Galindo, A.A. (2016) Caracterización de modelos de digestión humana a partir de sus representaciones y análisis de su evolución en un grupo de docentes y auxiliares académicos. Enseñanza de las Ciencias: revista de investigación y experiencias didácticas 34 (1), 129-147. Doi: 10.5565/rev/ensciencias.1748

Bahar, M., Ozel, M., Prokop, P., Usak, M. (2008) Science students teachers' ideas of the heart. Journal of Baltic Science Education 7 (2), 78-85.

Buckley, B.C. (2000). Interactive multimedia and model-based learning in biology. International Journal of Science Education 22 (9), 895-935.

Biasutto, S.N., Caussa, L.I., Criado del Río, L.E. (2006) Teaching anatomy: Cadavers vs. computers? Annals of Anatomy 188 (2), 187-190. Doi: 10.1016/j.aanat.2005.07.007

Bowen, T. (2017) Assessing visual literacy: a case study of developing a rubric for identifying and applying criteria to undergraduate student learning. Teaching in Higher Education, 115. Doi: 10.1080/13562517.2017.1289507

Brenton, H., Hernandez, J., Bello, F., Strutton, P., Purkayastha, S., Firth, T., Darzi, A. (2007) Using multimedia and Web3D to enhance anatomy teaching. Computers and Education 49 (1), 32-53. Doi:10.1016/j.compedu.2005.06.005

Carvalho G.S., Silva R., Clément, P. (2007) Historical analysis of Portuguese primary school textbooks (1920-2005) on the topic of digestion. International Journal of Science Education 29 (2), 173-193. Doi: 10.1080/09500690600739340

Cheng Y-L., Mix K.S. (2014) Spatial Training Improves Children's Mathematics Ability. Journal of Cognition and Development 15 (1), 2-11. Doi: 10.1080/15248372.2012.725186

Coleman, J.M., McTigue, E.M., Smolkin, L.B. (2011) Elementary teachers' use of graphical representations in science teaching. Journal of Science Teacher Education 22 (7), 613-643. Doi: $10.1007 /$ s10972-010-9204-1

Davies, D. (2010) Teaching science creatively. New York: Routledge.

De Jager, T. (2017) Perceived advantages of 3D lessons in constructive learning for South African student teachers encountering learning barriers. International Journal of Inclusive Education 21 (1), 90-102. Doi:10.1080/13603116.2016.1184329

Dimopoulos, K., Koulaidis, V., Sklaveniti, S. (2003) Towards an analysis of visual images in school science textbooks and press articles about science and technology. Research in Science Education 33 (2), 189-216. Doi: 10.1023/A:1025006310503

Donovan, J., Venville, G. (2014) Blood and bones: The influence of the mass media on Australian primary school children's understandings of genes and DNA. Science \& Education 23 (2), 325-360. Doi:10.1007/s11191-012-9491-3

Eilam, B. (2012) Teaching, learning, and visual literacy. The dual role of visual representation. Cambridge: Cambridge University Press.

Fančovičova, J., Prokop, P. (2014) The effects of 3D plastic models of animals and cadaveric dissection on students' perceptions of the internal organs of animals. Journal of Baltic Science Education 13 (6), 767-775. Recuperado de: http://www.scientiasocialis.lt/jbse/? $\mathrm{q}=$ node $/ 397$

Felten, P. (2008) Visual literacy. Change: The Magazine of Higher Learning 40 (6), 60-64. Doi: 10.3200/CHNG.40.6.60-64

Field, A.P. (2009) Discovering statistics using SPSS, Third edition. London: SAGE. 
Freeman, S., Eddy, S.L., McDonough, M., Smith, M.K., Okoroafor, N., Jordt, H., Wenderoth, M.P. (2014). Active learning increases student performance in science, engineering, and mathematics. Proceedings of the National Academy of Sciences, 111 (23), 8410-8415. Recuperado de : www.pnas.org/cgi/doi/10.1073/pnas.1319030111

García Fernández, B., Ruiz-Gallardo, J. R. (2017) Visual Literacy in Primary Science: Exploring Anatomy Cross-Section Production Skills. Journal of Science Education and Technology 26 (2), 161-174. Doi:10.1007/s10956-016-9662-0

Gatt, S., Saliba, M. (2006) Young children's ideas about the heart. Proceedings of the $3^{\text {rd }}$ International Conference on Hands-On Science, pp. 17-23. Vila Verde, Portugal: Universidade do Minho.

Gil Flores, J. (2014). Metodologías didácticas empleadas en las clases de ciencias y su contribución a la explicación del rendimiento. Revista de Educación 366, 190-214. Doi: 10.4438/1988-592X-RE-2014-366-271

Harrison, A.G., Treagust, D.F. (2000) A typology of school science models. International Journal of Science Education 22 (9), 1011-1026. Doi:10.1080/095006900416884

Jaipal, K. (2010) Meaning Making Through Multiple Modalities in a Biology Classroom: A Multimodal Semiotics Discourse Analysis. Science education 94, 48-72. Doi:10.1002/sce.20359

Justi, R.S., Gilbert, J.K. (2002) Science teachers' knowledge about and attitudes towards the use of models and modelling in learning science. International Journal of Science Education 24 (12), 1273-1292. Doi:10.1080/09500690210163198

Kress. G., Jewitt, C., Ogborn, J., Charalampos, T. (2001) Multimodal Teaching and Learning: The Rhetorics of the Science Classroom. New York: Continuum.

López-Manjón, A., Postigo, Y. (2014) Análisis de las imágenes del cuerpo humano en libros de texto españoles de primaria. Enseñanza de las Ciencias 32 (3), 551-570.

Doi:10.5565/rev/ensciencias.1319

López-Manjón, A. Postigo, Y. (2016) ¿Qué libro de texto elegir? La competencia visual en las actividades con imágenes. Revista Eureka sobre Enseñanza y Divulgación de las Ciencias 13 (1), 84-101. Recuperado de: http://hdl.handle.net/10498/18016

Martínez-Chico, M., Jiménez Liso, M.R., López-Gay, Lucio-Villegas, R. (2015) Efecto de un programa formativo para enseñar ciencias por indagación basada en modelos, en las concepciones didácticas de los futuros maestros. Revista Eureka sobre Enseñanza y Divulgación de las Ciencias 12 (1), 149-166.

McTigue, E.M., Flowers, A.C. (2011) Science visual literacy: learners' perceptions and knowledge of diagrams. The Reading Teacher 64 (8), 578-589. Doi: 10.1598/RT.64.8.3

National Academies of Sciences, Engineering, and Medicine (2016) Science Literacy: Concepts, Contexts, and Consequences. Washington, D.C.: The National Academies Press. Doi: $10.17226 / 23595$

National Research Council (2012) In: Schweingruber H, Keller T, Quinn H (eds) A framework for K-12 science education: practices, crosscutting concepts, and core ideas. Washington: National Academies Press. Recuperado de: https://www.nap.edu/catalog/13165/a-frameworkfor-k-12-science-education-practices-crosscutting-concepts 
National Research Council (2013) The next generation science standards, appendix F: science and engineering practices. United States: National Academy of Science. Recuperado de: http://www.nextgenscience.org/next-generation-science-standards

Núñez, F., Banet, E. (1997) Students' conceptual patterns of human nutrition. International Journal of Science Education 19 (5), 509-526. Doi: 10.1080/0950069970190502

Pérez de Eulate González, L., Llorente, E., Andrieu, A. (1999) Las imágenes de digestión y excreción en los textos de primaria. Enseñanza de las ciencias 17 (2), 165-178. Recuperado de: http://www.raco.cat/index.php/Ensenanza/article/view/21571

Postigo, Y., López-Manjón, A. (2012) Students' conceptions of biological images as representational devices. Revista Colombiana de Psicología 21 (2), 265-28.

Postigo, Y., López-Manjón, A. (2015) Graphicacy in biology textbooks: analysis of activities with images/Alfabetización gráfica en libros de texto de biología: análisis de las actividades con imágenes. Infancia y Aprendizaje 38 (3), 509-541. Doi: 10.1080/02103702.2015.1054667

Prain, V., Waldrip, B. (2006) An exploratory study of teachers' and students' use of multimodal representations of concepts in primary science. International Journal of Science Education 28 (15), 1843-1866. Doi: 10.1080/09500690600718294

Preece, D., Williams, S.B., Lam, R., Weller, R. (2013) "Let's get physical": advantages of a physical model over 3D computer models and textbooks in learning imaging anatomy. Anatomical sciences education 6 (4), 216-224. Doi: 10.1002/ase.1345

Real Decreto 126/2014, de 28 de febrero, por el que se establece el currículo básico de la Educación Primaria. (2014). Boletín Oficial del Estado 52, 19349-19420.

Ruiz-Gallardo, J.R., García Fernández, B., Mateos Jiménez, A. (2017) Visual Literacy in Preservice Teachers: a Case Study in Biology. Research in Science Education, 1-23. Doi: $10.1007 / \mathrm{s} 11165-017-9634-2$

Ruiz-Gallardo, J.R., Valdés, A., Sáez, J.M., Roldán, J. (2012) La realización de gráficos como medio de aprendizaje en Biología. En J.M. Domínguez-Castiñeiras (Ed.) XXV Encuentro de Didáctica de las Ciencias Experimentales, libro de actas (pp. 1307-1312). Santiago de Compostela: Universidade de Santiago de Compostela.

Rybarczyk, B. (2011) Visual literacy in biology: a comparison of visual representations in textbooks and journal articles. Journal of College Science Teaching 41 (1), 106. Recuperado de: www.jstor.org/stable/43748290 